BULL. AUSTRAL. MATH. SOC.

$45 P 05,45 N 05,45 M 99$

VOL. 35 (1987) 275-289

\title{
NONLINEAR INTEGRAL OPERATORS \\ AND CHAOS IN BANACH SPACES
}

\author{
Phil Diamond
}

\begin{abstract}
Sufficient conditions are given for chaotic behaviour of continuous transformations on Banach spaces. The conditions avoid the requirement that mappings be expanding on compact sets and are probably easier to verify for many classes of operator equations than existing criteria. Two classes of integral operators on $C[0,1]$ are considered in the light of these results: one nonlinear but compact, the second noncompact.
\end{abstract}

\section{Introduction}

The study of chaotic dynamical behaviour on Banach spaces is relatively recent. Only a few theoretical papers have appeared which deal with the genuinely infinite dimensional case, although it is wellknown that the centre manifold theorem implies that many infinite dimensional processes have finite dimensional invariant attractors [9]. The self-reproductive cell PDE generates a chaotic semiflow on $C[0,1]$ ([7], [1], [2]), but this analysis is for a specific model, albeit surprisingly linear. Zaslavskii [11] has given general sufficient conditions involving "strong recursion structures" which depend on the character of the Frechet derivative of a Banach space mapping. Kloeden [5] describes wide sufficient conditions which are formally alike the

Received 15 April 1986.

Copyright Clearance Centre, Inc. Serial-fee code: $\infty 00-9727 / 87$ $\$ A 2.00+0.00$. 
reasonably well-understood finite dimensional result [4].

Unfortunately, the criteria of these last two authors are hard to check and examples are difficult to come by. Zaslavskii provides a model with a strong recursion structure, but it is finite dimensional. On the other hand, kloeden requires that a map be expanding on a compact set. It is not always easy to demonstrate expansivity of integral operators in, for example, the sup norm on $C[0,1]$, while compact sets are not always natural objects of study within the context of noncompact operators. Here these conditions are weakened to a point where they can be more readily verified. The theorems are applied to nonlinear compact operators of the form $T:=(T x)(t)=\int_{0}^{1} \frac{r+t}{p+t+s} \psi(s) m(x(s)) d s$. A nonlinear and generally noncompact class of the form $\left.L x K x:=(T x)(t)=(L x)(t) \int_{0}^{1} \frac{r+t}{p+t+s} m(x)(s)\right) d s$ where $L$ is affine, is also discussed. Although the theorems appear to be only mild generalisations of other results, their virtue lies in the fact that they can actually be applied to mappings such as those above.

The following section 2 sets out definitions and states results. corollaries to theorems 2 and 3 provide classes of chaotic mappings. The theorems are proved in section 3 and the examples discussed in sections 4 and 5 .

\section{Definitions, notations and results}

Denote by $C_{+}$the cone of non-negative functions in $C[0,1]$ and write $S(\alpha ; \varepsilon)=\{x \in C[0,1]:|| x-\alpha \| \leq \varepsilon\}$. For $\alpha, \beta \in C_{+}$and $\alpha \leq \beta$ (that is $\beta-\alpha \in C_{+}$), define the $\alpha, \beta$-slice $D\{\alpha, \beta\}=\left\{x \in C_{+}\right.$: $\alpha \leq x \leq \beta\}$. Suppose $T$ maps $C_{+}$to itself. If for $u \in D\{\alpha, \beta\}$ there exists $v \in D\{\alpha, \beta\}$ such that $u=T v$, we say that $u$ has a $T$-representation in $D\{\alpha, \beta\}$. If $\alpha, \beta$ have $T$-representations, define the slice

$$
D^{T}\{\alpha, \beta\}=\{x \in D\{\alpha, \beta\}: x \text { has a } T \text {-representation }\}
$$

and when there is no ambiguity use the abbreviations $D, D^{T}$.

If $X=D^{T}\{\alpha, \beta\}, \quad a \in X$ and $0<\lambda<1$ define 


$$
\begin{aligned}
\tilde{X}(a, \lambda)= & \{x \in X:|x(t)-a(t)| \geq \lambda|| x-a||, 0 \leq t \leq 1, \\
& \text { and either } \left.x-a \in C_{+} \text {or } a-x \in C_{+}\right\}
\end{aligned}
$$

Clearly $\tilde{X}$ is closed, but possibly empty.

DEFINITION 1. Let $T: C[0,1] \rightarrow C[0,1]$ be a continuous map and $A$ be a closed bounded set in $C_{+}, T$ is said to be partially expansive on $A$ if $T$ has a fixed point $a \in A$ and there exists $\lambda \in(0,1)$ and $\mu>1$ such that $\tilde{A}(a, \lambda)$ is nonempty and $|(T x)(t)-a| \geq \mu|| x-a||$ for each $x \in \tilde{A}(a, \lambda)$ and all $t \in[0,1]$.

DEFINITION 2. Let $X$ be a Banach space and $A$ be a bounded subset of $X$. Following Kuratowski [6] define $\gamma(A)$, the measure of noncompactness of $A$, to be inf $\{\delta>0: A$ can be covered by a finite number of sets of diameter not greater than $\delta\}$. Suppose $T$ maps $X$ continuously into itself and that $T$ takes bounded sets to bounded sets. If, for some $k \in[0,1), \gamma(T(A)) \leq k \gamma(A)$ for every bounded subset $A$ of $X$, we say that $T$ is a strict set contraction. Analogously, if $\gamma(T(A)) \geq k \gamma(A)$ for every bounded subset $A$ of $X$ and some fixed $k>1$ we say that $T$ is a strict set dization. If $T$ is a strict set dilation and $T^{-1}$ exists, then $T^{-1}$ is a strict set contraction.

The first result uses ideas of leggett [8] and shows that the noncompact maps $L x K x$ are in some cases strict set dilations.

THEOREM 1. Let $A$ be a subset of the Banach algebra $B$ and suppose that $T: A \rightarrow B$ is of the form $T x=(L x)(K x)$ where (i) $L: A \rightarrow B$ satisfies $|(L x)(t)-(L y)(t)| \geq \mu|| x-y||$ for some $\mu>0$, each $t \in[0,1]$ and all $x, y \in A ;$ and

(ii) $K: A \rightarrow B$ is compact

Suppose $v=\inf ((K x)(t): x \in A, 0 \leq t \leq 1\}>0$. Then if $\mu \nu>1, T$ is a strict set dilation. If, additionally, $T$ has a fixed point $a \in A$ and $\mu \nu-\| L a|||| K||>1$, then $T$ is partially expansive on $A$.

DEFINITION 3. Let $T: X+X$ be a continuous mapping of the Banach space $X$ and suppose there exist nonempty closed bounded subsets $A, B$ 
of $X$ and integers $n_{1}, n_{2} \geq 1$ such that

(1) $B \subseteq A \subseteq T(A)$, and $T$ is injective on $A$;

(2) $T^{n}(B) \cap A=\varnothing$;

(3) $T^{n_{1}+n_{2}}$ is injective on $B$ and $T^{n_{1}+n_{2}}(B) \geq A$.

Then we say that $T \in C H(X)$. In the particular case $X=\mathbb{R}$, replace $A$ and $B$ by the compact intervals $I, J$ respectively.

Kloeden [4], [5] has shown that if $T \in C H(X)$, that if $A$ and $B$ are compact and $T$ expanding on $A$, and if $A$ is convex, then $T$ is chaotic (see [4] for a definition of chaos). Theorem 1 will be applied to show the following theorems and corollaries which generalise kloeden's result and demonstrate classes of chaotic operators.

THEOREM 2. Let $X$ be a Banach space and $T$ a continuous mapping from $X$ to itself. Suppose that

(1) $T \in C H(X)$;

(2) $T$ is partially expansive on the set $A$ of Definition 3 ;

(3) The sets $A, B$ of Definition 3 are compact, and $A$ is convex.

Then $T$ is a chaotic mapping on $X$.

COROLLARY 2. Let the mapping $T: C[0,1] \rightarrow C[0,1]$ be defined by

$$
(T x)(t)=\int_{0}^{1} \frac{r+t}{x+t+s} \psi(s) m(x(s)) d s \text {, }
$$

where $r>0, \psi$ is a positive continuous function and $m:[0,1] \rightarrow[0,1]$ is continuous and such that $m \geq 0$ and

(1) $m \in C H[0,1]$;

(2) $m$ is expanding on the interval $I$ of Definition 3;

(3) $m$ is strictly monotone on $I$.

Then there exist $r$ and $\psi$ for which $T$ is chaotic on $C_{+}$. 
THEOREM 3. Let $X$ be a Banach space and $T$ a mapping from $X$ to itself. Suppose that

(1) $T \in C H(X)$

(2) $T$ is a strict set dilation on, and partially expansive on, the set $A$ of Definition 3;

(3) $A$ is convex.

Then $T$ is a chaotic mapping on $X$.

COROLLARY 3. Let the mapping $T: C[0,1] \rightarrow C[0,1]$ be defined by

$$
(T x)(t)=\ell(1-x(t)) \int_{0}^{1} \frac{r+t}{x+t+s} \psi(s) m(x(s)) d s,
$$

where $r>0, \psi$ is a positive continuous function, $\ell$ is a positive real and $m:[0,1] \rightarrow[0,1]$ is continuous and such that $m \geq 0$ and

(1) $M:=\ell(1-t) m(t) \in C H[0,1] ;$

(2) $M$ is expanding on the interval I of Definition 3 ;

(3) $m$ is strictly monotonic on $I$;

(4) $\inf _{t \in I} m(t)>\sup _{t \in I}(1-t)$.

Then there exist $\ell, r, \psi$ for which $T$ is chaotic on $C_{+}$.

\section{Proofs of theorems}

Proof of Theorem 1. Let $C$ be a bounded subset of $A$, let $\varepsilon>0$ and set $\omega=\sup _{u \in C} \||L u| \mid$. Since $K(C)$ is relatively compact, there exist finitely many sets $E_{1}, E_{2}, \ldots, E_{k}$ in $B$ such that diam $E_{i}<3 \varepsilon / \varepsilon \omega$, $1 \leq i \leq k$ and $K(C)=\underset{i=1}{k} E_{i}$. Sets $C_{1}, C_{2}, \ldots, C_{n}$ may be so chosen that diam $c_{i} \geq \gamma(C)+\varepsilon / 2 \mu \nu$ for at least one $i=i^{*}, 1 \leq i^{*} \leq n$, and $C=\stackrel{\cup}{\underline{U}}_{1} C_{i}$. Define $S_{i j}=L\left(C_{i}\right) E_{j}, i=1, \ldots, n, j=1, \ldots, k$ and note that $T(C)$ is covered by the $S_{i j}$. If $w, z \in S_{i * j}$, then there exist $u, v \in C_{i *}$ and $x, y \in E_{j}$ such that $w=x L u$ and $z=y L v$. Then 


$$
\begin{aligned}
|w(t)-z(t)| & =|(x L u)(t)-(y L u)(t)+(y L u)(t)-(y L v)(t)| \\
& \geq|(y L u)(t)-(y L v)(t)|-|(x L u)(t)-(y L u)(t)| \\
& =|y(t)((L u)(t)-(L v)(t))|-|(x(t)-y(t))(L u)(t)| \\
& =|y(t)||(L u)(t)-(L v)(t)|-|(L u)(t)||x(t)-y(t)| \\
& \geq v|(L u)(t)-(L v)(t)|-|| L u|||| x-y|| \\
& \geq v|(L u)(t)-(L v)(t)|-3 \varepsilon / 2
\end{aligned}
$$

Thus ||$w-z||+3 \varepsilon / 2 \geq v|| L u-L v|| \geq \mu \nu|| u-v||$ and it follows that diam $S_{i * j} \geq \mu \nu \gamma(C)-\varepsilon$. Thus $\gamma(T(C)) \geq \mu \nu \gamma(C)$ and $T$ is a strict set dilation. Also, $|(K x L x)(t)-a(t)|=|(K x L x)(t)-(K a L a)(t)|$ $\geq|(K x)(t)||(L x)(t)-(L a)(t)|-|(L a)(t)||(K x)(t)-(K a)(t)| \geq \mu \nu|| x-a||$ -||$K|||| L a|||| x-a||$, and $T$ is partially expansive.

Proof of Theorem 2. The much weaker condition of partial expansivity replaces the requirement that $f$ be strictly expanding - otherwise the conditions are those of [4], [5]. Consequently, all the chaotic properties follow from the usual constructions, except that it remains to show the

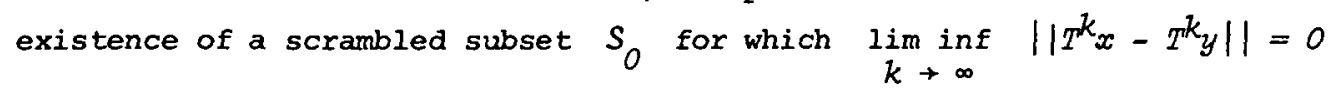
for all $x, y \in S_{O}$. This is a consequence of the following lemma.

LEMMA 2a. Under the conditions of Theorem 2, given $\varepsilon>0$ there exists a positive integer $\tau(\varepsilon)$ and a nonempty closed subset $\tilde{E}$ of $B$ such that $T_{A}^{-k}(\tilde{E}) \subset A \cap S(a ; \varepsilon)$ for all $k \geq \tau(\varepsilon)$, where $a \in A$ is $a$ fixed point of $T$.

Proof. Since $T^{n_{1}+n_{2}}(B) \supseteq A$, by the continuity of $T$ there exists a nonempty compact subset $E$ of $B$ such that $T^{n_{1}+n_{2}}(E)=A$. From (1) of Definition 3 there exists a continuous inverse $T_{A}^{-1}: A \rightarrow A$ and so by the Schauder fixed point theorem there is $a \in A$ with $T_{A}^{-1} a=a$, that is $T a=a$. From the definition of partial expansivity there exists $\lambda \in(0,1)$ such that $|(T x)(t)-a| \geq \mu|| x-a||, \mu>1$, for all 
$x \in \tilde{A}(a, \lambda)$. Now $\tilde{A}$ is closed in $A$, hence compact. Denote by $\tilde{T}_{A}^{-1}$ the restriction of the inverse to $\tilde{A}$, which is continuous on $\tilde{A}$. Then for any $x \in \tilde{E}(a, \lambda) \subset \tilde{A},|| \tilde{T}_{A}^{-k} x-a|| \leq \mu^{-k}|| x-a||$ for each integer $k \geq 1$. Hence for any $\varepsilon>0$ there exists an integer $j=j(x, \varepsilon)$ such that

$$
\tilde{T}_{A}^{-j}(x) \in \tilde{A} \cap S(a ; \varepsilon) \subset A \cap S(a ; \varepsilon)
$$

From continuity there exists $\delta=\delta(x, \varepsilon)>0$ such that

$$
\tilde{T}_{A}^{-j}(\tilde{A} \cap \text { int } S(x, \delta)) \subset A \cap S(a ; \varepsilon) .
$$

The collection $\{$ int $S(x ; \delta): x \in \tilde{E}$ \} is an open cover of $\tilde{E}$. It is easy to see that $\tilde{E}$ is closed in $\tilde{A}$, so compact, and thus there is a finite subcover $\left\{\right.$ int $\left.S\left(x_{i} ; \delta_{i}\right): 1 \leq i \leq n\right\}$. Let $\tau(\varepsilon)=\max _{1 \leq i \leq n} j\left(x_{i}, \varepsilon\right)$ and note that $\tilde{T}_{A}^{-\tau}(x) \in A \cap S(a ; \varepsilon)$ for all $x \in \tilde{E}$. From relation (3) $\tilde{T}_{A}^{-k}(\tilde{E}) \subset A \cap S(a ; \varepsilon)$ for all $k \geq \tau(\varepsilon)$.

Proof of Theorem 3. As in Theorem 2, most of the construction of [4] goes through. Only two things need to be checked vis-a-vis the weakened conditions : first that strict set dilations on closed convex sets provide the fixed points needed by the construction; secondly that an analogue of Lemma $2 a$ holds so that $S_{O}$ exists. These are the substance of the following two lemmas.

LEMMA 3a. Let $g$ be the continuous inverse of $T^{n_{1}+n_{2}}$ on $A$ and let $T_{A}^{-1}$ be the continuous inverse of $T$ on $A$. Then for each integer $k \geq 0, T_{A}^{-k} \circ g: A \rightarrow A$ has a fixed point $y_{k} \in A$.

Proof. Since $T$ is a strict set dilator, $T_{A}^{-1}$ is a strict set. contractor, and hence so are $T_{A}^{-k}$ and $g$. A theorem of Darbo [3] states : if a strict set contraction $f$ leaves invariant a closed, bounded convex subset $C$ of a Banach space, then $f$ has a fixed point in $C$. Thus $T_{A}^{-k} \circ g$ has a fixed point in $A$. 
LEMMA 3b. Under the conditions of Theorem 3 , given $\varepsilon>0$ there exists a positive integer $\tau=\tau(\varepsilon)$ and a nonempty closed bounded subset $\tilde{E}$ of $B$ such that $T_{A}^{-k}(\tilde{E}) \subset A \cap S(a ; \varepsilon)$ for all $k \geq \tau(\varepsilon)$.

Proof. Since $T^{n_{1}+n_{2}}(B) \supseteq A$, there exists a nonempty closed bounded subset $E$ of $B$ such that $T^{n_{1}+n_{2}}(E)=A$. Py Darbo's theorem there is a fixed point $a \in \tilde{A}$ of the inverse $\tilde{T}_{A}^{-1}$ of $T$ on $\tilde{A}$. As in Lemma 2a, it follows that $\tilde{T}_{A}^{-k} x \rightarrow a$ as $k \rightarrow \infty$ for all $x \in \tilde{E}(a, \lambda) \subset \tilde{A}$. Hence for any $\varepsilon>0$ there exists an integer $j=j(x, \varepsilon)$ such that $\tilde{T}_{A}^{-j}(x) \in A \cap S(a ; \varepsilon) \subset A \cap S(a ; \varepsilon)$. From continuity there exists $\delta=\delta(x, \varepsilon)>0$ such that

$$
\tilde{T}_{A}^{-j}(\tilde{A} \cap \text { int } S(x ; \delta)) \subset A \cap S(a ; \varepsilon)
$$

The collection $\{$ int $S(x ; \delta): x \in \tilde{E}\}$ is an open cover of $\tilde{E}$. Consequently $T_{A}^{-j}($ int $S(x ; \delta))$ is an open cover of $\tilde{T}_{A}^{-j} \tilde{E}$. So $\delta$ may be chosen so small that

$$
\begin{aligned}
\operatorname{diam} T^{-j}(\operatorname{int} S(x ; \delta)) & \leq \gamma\left(\tilde{T}^{-j} \tilde{E}\right)+\varepsilon / 2 \\
& \leq \rho^{-j} \gamma(\tilde{E})+\varepsilon / 2
\end{aligned}
$$

where $\rho>1$ is the constant of strict set dilation. Let $\tau=\tau(\varepsilon)$ $=\min \left\{j: \rho^{-j} \gamma(\tilde{E})<\varepsilon / 2\right\}$. Then $\tilde{T}_{A}^{-\tau}(x) \in S(a ; \varepsilon) \cap A$ for all $x \in \tilde{E}$. That is, $\tilde{T}_{A}^{-k}(\tilde{E}) \subset A \cap S(a ; \varepsilon)$ for all $k \geq \tau(\varepsilon)$.

\section{Proof of Corollary 2}

A general proof involves a daunting number of constants depending on $\int_{0}^{1} \psi$, distances between the end points of the intervals $I$ and $J$, distances between slices in $C$, and on $n_{1}+n_{2}$. However all the ideas of the proof are clearly displayed in the following specific example. 
COROLLARY $2^{\prime}$ Let $H(t)=\left\{\begin{array}{cc}2 t, & 0 \leq t \leq 1 / 2, \\ 2-2 t, & 1 / 2 \leq t \leq 1 .\end{array}\right.$ Then there

exist a positive constant constant $r$ and a positive continuous function $\psi$ such that the mapping $T: C[0,1] \rightarrow C[0,1]$ defined by

$$
(T x)(t)=\int_{0}^{1} \frac{r+t}{r+t+s} \psi(s) H(x(s)) d s
$$

is chaotic.

A series of Lemmas (Lemma $2 \cdot j, 1 \leq j \leq 5$ ) will show that the operator (4) satisfies the conditions of Theorem 2. At one point only (Note 2.3) will it be necessary to indicate how and why anything extra need be done to extend the treatment to the operator defined by (1).

The following notation is used throughout the remainder of this section : $\psi(s, t)=(p+t) \psi(s) /(x+t+s)$,

$$
\begin{aligned}
& \phi:=\phi(t, r)=\int_{0}^{1} \frac{r+t}{x+t+s} \psi(s) d s, \quad p=1+q=\int_{O}^{1} \psi(s) d s, \\
& q_{1}=(q r-1) /(r+1), \quad p_{1}=p r /(r+1), A=D^{T}\{17 \phi / 32,7 \phi / 8\} . \\
& B=D^{T}\{3 \phi / 4,7 \phi / 8\}, \quad \alpha=17 \phi / 32, \quad \beta=7 \phi / 8 .
\end{aligned}
$$

Note that $p_{1} \leq \phi \leq p$ for all $r \geq 0$.

LEMMA 2.1. The slices $D^{T}\{\alpha, \beta\}$ are compact convex sets.

Proof. Let $x, y \in D^{T}\{\alpha, \beta\}$, and $n \in[0,1]$. Clearly $\alpha \leq n x+(1-n) y \leq \beta$. Since $x, y$ are T-representations there are elements $u_{x}, u_{y} \in D\{\alpha, \beta\} \quad$ such that $x=T u_{x}, y=T u_{y}$ and so $n x+(1-n) y=\int_{0}^{1} \psi(s, t)\left(n H\left(u_{x}(s)\right)+(1-n) H\left(u_{y}(s)\right)\right) d s$. Since $p_{1} \leq|| \phi|| \leq p$, for suitable $r, \psi[|| \alpha||, \| \beta||] \subset[1 / 2,1]$ and so $n H\left(u_{x}\right)+\left(1-n B\left(u_{y}\right)=n\left(2-2 u_{x}\right)+(1-n)\left(2-2 u_{y}\right)=H\left(n u_{x}+(1-n) u_{y}\right)\right.$, so $n x+(1-N)$ is also a $T$-representation and thus in $D^{T}\{\alpha, \beta\}$. For $t, t^{\prime} \in[0,1]$, 


$$
x(t)-x\left(t^{2}\right)=\int_{0}^{1} \frac{\left(t^{\prime}-t\right) \psi(s, t)}{\left(x^{2}+t+s\right)\left(p+t^{\prime}+s\right)} H\left(u_{x}(s)\right) d s
$$

and so the slice $D^{T}\{\alpha, \beta\}$ is equicontinuous since $\left\|H \circ u_{x}\right\| \leq 2-2\|\alpha\| \mid$.

NOTE 2.1. For the general mapping (1), define $\alpha_{1}, \beta_{1}$ appropriately so that $\left[\left\|\alpha_{1}\right\|,\left\|\beta_{1}\right\|\right] \subset I$. Then $m$ is injective on $\left[\left\|\alpha_{1}\right\|,\left\|\beta_{1}\right\|\right]$ and there exists $v_{x, y} \in D\left[\alpha_{1}, B_{1}\right]$ such that $m\left(v_{x, y}(t)\right)=\eta m\left(u_{x}(t)\right)$ $+(1-n) m\left(u_{y}(t)\right)$ and this extends the convexity argument of Lemma 2.1 to the general case.

LEMMA 2.2. There exist a positive constont $r$ and a positive continuous fronction $\psi$ such that

$$
A \subset T(A), \quad T(B) \cap A=\emptyset \text { and } T^{2}(B) \supset A \text {. }
$$

Proof. Define $A_{-}=D^{T}\left\{17 p / 32,7 p_{1} / 8\right\} \subset A \subset A_{+}=D^{T}\left\{17 p_{1} / 32,7 p / 8\right\}$,

$$
B_{-}=D^{T}\left\{3 p / 4,7 p_{1} / 8\right\} \subset B \subset B_{+}=D^{T}\left\{3 p_{1} / 4,7 p / 8\right\} \text {. }
$$

It will be shown that $T\left(A_{-}\right) \supset A, T\left(B_{+}\right) \cap A=\varnothing$ and $T^{2}\left(B_{-}\right) \supset A$. We say that $T$ is isotone on a slice if $x_{1} \leq x_{2}$ implies that $T x_{1} \leq T x_{2}$, and that $T$ is anti-isotone if $x_{1} \leq x_{2}$ implies that $T x_{1} \geq T x_{2}$. Now $T \quad$ is anti-isotone on all the sets $A_{ \pm}, B_{ \pm}$for $1 \leq p \leq 8 / 7$ and $r$ sufficiently large. Moreover, every element of $T\left(A_{\underline{t}}\right), T\left(B_{\underline{ \pm}}\right)$ has a T-representation and so it suffices to consider only the images under $T$ of the "end-functions" of each slice (provided $T$ is injective, see Lemma 2.3). Suppose $\psi$ is so chosen that $0<q \leq 1 / 35$, and that $r \geq 48$. Then

$$
\begin{aligned}
(T 17 p / 32)(t) & =\int_{0}^{1} \psi(s, t)(2-17(1+q) / 16) d s \\
& =(15 / 16-17 q / 16) \phi \\
& >29 \phi / 32 \text { since } q \leq 1 / 35, \text { and }
\end{aligned}
$$




$$
\begin{aligned}
\left(T 7 p_{1} / 8\right)(t) & =(1 / 4+7 / 4(r+1)-7 q r / 4(r+1) \phi \\
& <\phi / 2 \text { since } r \geq 48 .
\end{aligned}
$$

In much the same way $T\left(B_{+}\right) \cap A=\emptyset$, and $T^{2}\left(B_{+}\right) \supset A$ is only slightly more complicated because of the second iteration.

LEMMA 2.3. $T$ is injective on $A$ and $T^{2}$ is injective on $B$.

Proof. $T x=T y$ for all $x, y \in A$ if and only if $T_{0} x=T_{o} y$, where

$$
\left(T_{0} x\right)(t)=\int_{0}^{1} \frac{\psi(s) x(s)}{x+t+s} d s
$$

But (5) is the stieltjes transform of $u(t)=\left\{\begin{array}{cc}\psi(t)_{x}(t), & 0 \leq t \leq 1, \\ 0, & >1\end{array}\right.$,

translated by $r$, and is thus injective ([10], chapter VIII, Theorem $5 b)$.

Since $\psi$ is positive, $x=y$. On the other hand $T(B) \subset D^{T}\{0,1 / 2\}$

and since $T$ is injective on $B, T^{2} x=T^{2} y$ for all $x, y \in B$ if and only if $T_{o^{x}}=T_{\sigma^{y}}$ and the same argument prevails.

NOTE 2.3. In the general case $T_{0}$ has the form

$$
\left(T_{0} x\right)(t)=\int_{0}^{1} \frac{\psi(s) m(x(s))}{r+t+s} d s
$$

Suppose that $m$ is strictly monotonic on $I$ and that $D^{T}\{\alpha, \beta\}$ is such that $[|| \alpha||,\|\beta\|] \subset I$. For $x, y \in D^{T}\{\alpha, \beta\}, T_{o} x=T_{o} y \quad$ if and only if $m(x(t))=m(y(t))$ on $[0,1]$ by the injectiveness of the stieltjes transform. Since $m$ is strictly monotonic on $I, x=y$. To see that $T^{n_{1}+n_{2}}$ is injective on $B$, use the injectiveness of $m^{n_{1}+n_{2}}$ on $J$, the argument of Note 2.1 and the stieltjes transform.

LEMMA 2.4. There exists a fixed point a $\in A$ of $T$.

Proof. $T$ is injective and continuous on $A$, so there is a continuous inverse $T_{A}^{-1} A \rightarrow A$. Since $A$ is compact and convex the 
Schauder fixed point theorem may be applied and, any fixed point of $T_{A}^{-1}$ is also a fixed point of $T$.

LEMMA 2.5. Let $r, p$ be as in Lemma 2.2 and choose $\lambda>\left(2 p_{1}\right)^{-1}$. Then $\tilde{A}(a, \lambda)$ is a nonempty compact subset of $A$ and $T$ is partialzy expansive on $A$. Moreover $T(\tilde{A}) \supset \tilde{A}$.

Proof. Since $|\cdot|$ and $\|\cdot\|$ are continuous, it is obvious from the definition of $\tilde{A}$ that it is closed in $A$ and thus, by Lemma 2.1, compact. Moreover, if $x \in A$ then

$$
\begin{aligned}
|(T x)(t)-a(t)| & =|(T x)(t)-(T a)(t)| \\
& =2 \int_{0}^{1} \psi(s, t)|x(s)-a(s)| d s \quad \text { (see the definition of } \tilde{A} \text { ) } \\
& \geq 2 \lambda \phi(t)|| x-a||,
\end{aligned}
$$

these last two lines explicitly using the definition of $\tilde{A}(a, \lambda)$, and note that $\phi>p_{1}$ so take $\mu=2 \lambda p_{1}$. It remains to show that $\tilde{A}$ is nonempty. observe that $\operatorname{dist}(a, \partial A)>0$. For $\operatorname{TD}\{35 \phi / 64,13 \phi / 16\} \supset A$, so $\operatorname{dist}(a, \partial A) \geq p_{1} / 64$. It follows that there exists $u \in A, u \neq a$, since we may choose $v \in \tilde{C}_{+}(a, c)$ with $\left(2 p^{2}\right)^{-1}<c<1$ and ||$v-a|| \leq 1 / 64$; for then $u=T v \in A$ satisfies

$$
\begin{aligned}
|(T v)(t)-a(t)| & \geq c \phi(t)|| v-a|| \\
& \geq c p_{1}|| v-a||>\lambda|| v-a|| .
\end{aligned}
$$

\section{Proof of Corollary 3}

As in the previous section the ideas of the proof are more accessible in

COROLLARY $3^{\prime}$. There exist a positive constant $r$ and a positive continuous function $\psi$ such that the mapping $T: C[0,1] \rightarrow C[0,1]$ defined by

$$
(T x)(t)=3.9(1-x(t)) \int_{0}^{1} \psi(s, t) x(s) d s
$$


is chaotic.

Lemma 3.1 - 3.4 below show that (6) satisfies the conditions of Theorem 3, and Note 3.2 indicates the extension to the more general form (2). In addition to, and differing slightly from the notations of section 4, we say that $x$ has a $L K$-representation if it has the form $(L u)(K v)$ for $u, v \in D\{0,1\}$ and write $D^{L K}\{\alpha, \beta\}$ as the totality of $L K$-representations in $D\{\alpha, \beta\}$. In what follows $L u=3 \cdot g(1-u)$, $K v=\int_{0}^{1} \psi v, A=\overline{c o} D^{L K}\{\cdot 6 \phi, \cdot 85 \phi\} \quad$ (the closed convex hull of $D^{L K}$ ), $B=\overline{c o} D^{L K}\{\cdot 79 \phi ; \cdot 83 \phi\}, n_{1}=1$ and $n_{2}=3$. Note that in general the $D^{L K}$ are not compact.

LEMMA 3.1. There exist $r \in \mathbb{R}^{+}$and $\psi \in C_{+}$such that

$$
A \subset T(A), T^{2}(B) \cap A=\emptyset, T^{4}(B)>A \text {. }
$$

Proof. Define

$$
\begin{aligned}
& A_{-}=\overline{c O} D^{K L}\left\{\cdot 6 p, \cdot 85 p_{1}\right\} \subset A \subset \overline{c o} D^{K L}\left\{\cdot 6 p_{1}, \cdot 85 p\right\}=A_{+} \\
& B_{-}=\overline{c O} D^{K L}\left\{\cdot 79 p, \cdot 83 p_{1}\right\} \subset B \subset \overline{c o} D^{K L}\left\{\cdot 79 p_{1}, \cdot 83 p\right\}=B_{+}
\end{aligned}
$$

and we show that $T\left(A_{-}\right)>A_{,} T^{2}\left(B_{+}\right) \cap A=\varnothing$ and that $T^{4}\left(B_{-}\right) \supset A$ for suitable $r, \psi$. As in Lema 2.2, it suffices to consider only the images under $T$ of the end functions of each slice. Now $T$ is anti-isotone on $A_{ \pm}, B_{ \pm}$for $1 \leq p \leq(.85)^{-1}$, so $T(.6 p)=\left(.936-.468 q-1.404 q^{2}\right)_{\phi}$ $>\cdot 85 \phi$ provided $0<q<.13$, and $T\left(\cdot 85 p_{1}\right)=\left(.49725-.595 q_{1}\right.$ - $\left..7225 q_{1}^{2}\right) \phi<.6 \phi$ provided $q_{1} \geq 0$, that is $q<1 / r$. The other results follow in like, if more complicated, fashion.

LEMMA 3.2. Let $\psi$ be a positive continuous function and $r \in \mathbb{R}^{+}$, and define $T_{0} x=(1-x) K x$. Then $T_{0}$ is injective on $A$.

Proof. From Lemma 2.3, $K$ is injective on $D\{1 / 2,1\}$, so it suffices to consider the map $x \rightarrow x K x$. Now $x K x-y K y=(K y+x K)(x-y)$ and this can only be zero on appropriate slices $A$ if $x=y$. 
NOTE 3.2. (i) The calculations of Lemana 3.1 show that $T(B)$, $T^{2}(B)$ are subsets of $D\{1 / 2,1\}$, and $T^{3}(B)$ of $D\{0,1 / 2\}, T$ is injective on these images of $B$ and so $T^{4}$ is injective on $B$.

(ii) If $K$ has nonlinear kernel $m(x(s))$, then $x K x-y K y=(x-y) K y+x(K x-K y)$. As in Note 2.3 this is zero, on slices $D\{\alpha, \beta\}$ for which $m$ is strictly monotonic on $[|| \alpha||,\|\beta\|]$, if and only if $x=y$.

LEMMA 3.3. Let $r, p, q$ and $q_{1}$ be as in Lemma 3.1. Then $T$ is a strict set dizator on $A$.

Proof. The first part of Theorem 1 is satisfied with $\mu=3.9$ and $v=\inf \{(K x)(t): x \in D\{\cdot 65 \phi, \cdot 85 \phi\}, 0 \leq t \leq 1\} \geq .65 p_{1}^{2}$, $\mu \nu \geq 2 \cdot 535 p_{1}^{2}>1$.

LEMMA 3.4. There exists a fixed point $a \in A$ of $T$ and $T$ is partially expansive on $A$.

Proof. $T$ is continuous and injective on the closed convex set $A$ and so there is a continuous inverse $T_{A}^{-1}: A \rightarrow A$. But $T$ is a strict set dilator on $A$ and thus $T_{A}^{-1}$ is a strict set contractor. Again using Darbo's fixed point theorem (see Lemma 3a), $T_{A}^{-1} a=a$ for some $a \in A$. that is $T a=a$. From the observation that $T D\{\cdot 69 \phi, \cdot 80 \phi\}>A$, it follows that $a \in D\left\{\cdot 699_{,} \cdot 80 \phi\right\},|| L a|| \leq 3 \cdot 9 \sup _{0 \leq t \leq 1}(1-\cdot 69 \phi) \leq 3 \cdot 9\left(\cdot 31-\cdot 69 q_{1}\right)$. Since $\|K\| \leq p, \mu \nu-\|L a\|\|K\| \geq 2.535 p_{1}^{2}-1.209>1$, and $T$ is partially expansive by the second part of Theorem 1 . 


\section{References}

[1] P. Brunovsky, "Notes on chaos in the cell population partial differential equation", Nonlinear Anal. 7 (1983) 167-176.

[2] P. Brunovsky and J. Komornik, "Ergodicity and exactness of the shift on $C[0, \infty)$ and the semiflow of a first-order partial differential equation", J. Math. Anal. Appl. 104 (1984) 235-245.

[3] G. Darbo, "Punti uniti in transformazioni a codominio non compatto", Rend. Sem. Mat. Univ. Padova. 24 (1955), 84-92.

[4] P. Kloeden, "Chaotic difference equations in $\mathbb{I R}^{\mathrm{n}}$, J. Austral. Math. Soc. (Series A) 31 (1981), 217-225.

[5] P. Kloeden, "Cycles and chaos in higher dimensional difference equations" Proc. IXth Intemational Conference on Nonlinear Oscillations, ed. Yu. A. Mitropolsky, Kiev (1984).

[6] C. Kuratowski, Topologie, Vol. I, (Monografic Matematyczne No. 20, Warsaw, 1958).

[7] A Lasota, "Stable and chaotic solutions of a first-order partial differential equation", Nonlinear Anal. 5 (1981) 1181-1193.

[8] R.W. Leggett, "On certain nonlinear integral equations", J. Math. Anal. App Z. 57 (1977), 462-468.

[9] J. Mallet-Paret, "Negatively invariant sets of compact maps and an extension of a theorem of Cartwright", $J$. Differential Equations 22 (1976) 331-348.

[10] D.V. Widder, The Laplace Transform, (Princeton University Press, Princeton, N.J., 1946).

[11] B.G. Zaslavskii, "A quasihomoclinic structure generated by a semigroup of operators in Banach space", Siberian Math. J. 23 (1980) 825-833.

Mathematics Department

University of Queensland

St. Lucia

Queensland

Australia 